\title{
ON THE BEHAVIOR AT INFINITY OF SOLUTIONS TO DIFFERENCE EQUATIONS IN SCHRÖDINGER FORM
}

\author{
EVANS M. HARRELl II AND MANWAH LILIAN WONG
}

Abstract. We study the behavior at infinity of solutions of discrete Schrödinger equations. First we study pairs of discrete Schrödinger equations whose potential functions differ by a quantity that can be considered small in a suitable sense as the index $n \rightarrow \infty$. With simple assumptions on the growth rate of the solutions of the original system, we show that the perturbed system has a fundamental set of solutions with the same behavior at infinity, employing a variation-ofconstants scheme to produce a convergent iteration for the solutions of the second equation in terms of those of the original one.

Next, we present a sharp discrete analogue of the Liouville-Green (JWKB) transformation, making it possible to derive exponential behavior at infinity of a single difference equation, by explicitly constructing a comparison equation to which the perturbation results apply. After that we use the relations between the solution sets of two discrete Schrödinger equations differing by a perturbation to derive exponential dichotomy of solutions and to elucidate the structure of transfer matrices.

A final section contains illustrative examples, including some with large, oscillatory potentials, and an appendix discusses the connection between the discrete Schrördinger problem and orthogonal polynomials on the real line.

Mathematics subject classification (2010): Primary 34E10, 34L40, 39A06, 39A22; Secondary 39C41. Keywords and phrases: Difference equation, Schrödinger equation, Liouville-Green, JWKB, asymptotic equivalence, dichotomy, Green function, orthogonal polynomials.

\section{REFERENCES}

[1] R. P. Agarwal, Difference equations and inequalities, theory, methods and applications, Second Edition, Revised and Expanded, Marcel Dekker, New York, 2000, pp. 998.

[2] S. Agmon, Lectures on exponential decay, Princeton Univ. Press, Mathematical Notes 29. Princeton, 1982.

[3] Z. BenZAid AND D. A. LutZ, Asymptotic representation of solutions of perturbed systems of linear difference equations, Stud. Appl. Math. 77 (1987) 195-221.

[4] G. D. Birkhoff, General theory of linear difference equations, Trans. Amer. Math. Soc. 12 (1910) 243-284.

[5] P. A. BRAUN, WKB method for three-term recursion relations and quasienergies of an anharmonic oscillator, Teoret. Mat. Fiz. 37 (1978) 355-370, translated as Theoret. and Math. Phys. 37 (1978) 1070-1081.

[6] N. A. Chernyavskaya, Stability and absolute stability of a three-point difference scheme, Computers and Math. Appl. 45 (2003) 1181-1194.

[7] N. A. Chernyavs Kaya And L. Shuster, Necessary and sufficient conditions for solvability of the Hartman-Wintner problem for difference equations, J. Difference Eqns. Appl. 14 (2008) 1215-1254.

[8] C. V. Coffman, Asymptotic Behavior of Solutions of Ordinary Difference Equations, Trans. Amer. Math. Soc. 110 (1964) 22-51.

[9] E. B. Davies ANd E. M. Harrell II, Conformally flat Riemannian metrics, Schrödinger operators, and semiclassical approximation, J. Diff. Eqs. 66 (1987) 165-188. 
[10] M. S. P. EAstham, The asymptotic solution of linear differential systems. Applications of the Levinson Theorem, Oxford: Clarendon Press, 1989.

[11] J. S. Geronimo And D. T. Smith, WKB (Liouville-Green) Analysis of Second Order Difference Equations and Applications, J. Approx. Theory 69 (1992) 269-301.

[12] J. S. GERONImO AND W. VAN ASSCHE, Relative asymptotics for orthogonal polynomials with unbounded recurrence coefficients, J. Approx. Theory 62 (1990) 47-69.

[13] F. Gesztesy and B. Simon, The Xi Function, Acta Math. 176 (1994) 49-71.

[14] E. M. Harrell II AND M. L. Wong, On a transformation of Bohl and its discrete analogue, to appear in Proc. Symp. Pure Math.

[15] E. M. Harrell II And B. Simon, The Mathematical Theory of Resonances whose Widths are Exponentially Small, Duke Math. J. 47 (1980) 845-902.

[16] P. Hartman, Ordinary Differential Equations, Hartman, Baltimore, 1973.

[17] P. HISLOP AND I. M. SigAL, Introduction to spectral theory, with applications to Schrödinger operators, Springer Applied Mathematical Sciences 113. New York: Springer-Verlag, 1996.

[18] M. G. KREIN, On certain new studies in the perturbation theory for self-adjoint operators, in M. G. Krein, Topics in differential and integral equations and operator theory, I. Gohberg, ed. Birkhäuser, Basel, 1983, 107-172.

[19] N. LeVinson, The asymptotic nature of solutions of linear systems of differential equations, Duke Math. J. 15 (1948) 111-126.

[20] A. Máté, P. Nevai, V. Totik, Asymptotics for orthogonal polynomials defined on a recurrence relation, Constr. Approx. 1 (1985) 231-248.

[21] F. W. J. Olver, Asymptotics and Special Functions, New York: Academic, 1974.

[22] O. Perron, Über Stabilität und asymptotisches Verhalten der Lösungen eines Systems endlicher Differenzengleichungen, J. reine angew. Math. (Crelles Journal) 161 (1929) 41-64;

[23] H. PoInCARÉ, Sur les équations linéaires aux differentielles ordinaires et aux differences finies, Amer. J. Math. 7 (1885) 203-258.

[24] B. Simon, Orthogonal polynomials on the unit circle. Part 1: Classical Theory, AMS Colloquium Series, Amer. Math. Soc., Providence, RI, 2005.

[25] B. Simon, The Christoffel-Darboux kernel, Proc. Sympos. Pure Math., 79, Amer. Math. Soc., Providence, RI, 2008.

[26] D. T. SMITH, Exponential Decay of Resolvents of Banded Infinite Matrices and Asymptotics of Solutions of Linear Difference Equations, Georgia Institute of Technology, March, 1990.

[27] W. F. TRENCH, Linear perturbations of a nonoscillatory second order difference equation, J. Math. Analysis Appl. 273 (2002), 548-557.

[28] W. F. TRENCH, Linear perturbations of a nonoscillatory second order difference equation II, J. Math. Analysis Appl. 255 (2001), 627-635.

[29] M.-W. L. Wong, Generalized bounded variation and inserting point masses, Const. Approx. 30 (2009), 1-15.

[30] M.-W. L. Wong, Asymptotics of orthogonal polynomials and point perturbation on the unit circle, J. Approx. Theory 162 (2010), 1294-1321.

[31] M.-W. L. WonG, Asymptotic analysis of orthogonal polynomials via the transfer matrix approach, preprint, http://arxiv.org/abs/1009.1639v2 